\title{
Action Generation, Intention, and Agency in Motor and Body Awareness Deficits
}

University Press Scholarship Online

\section{Oxford Scholarship Online}

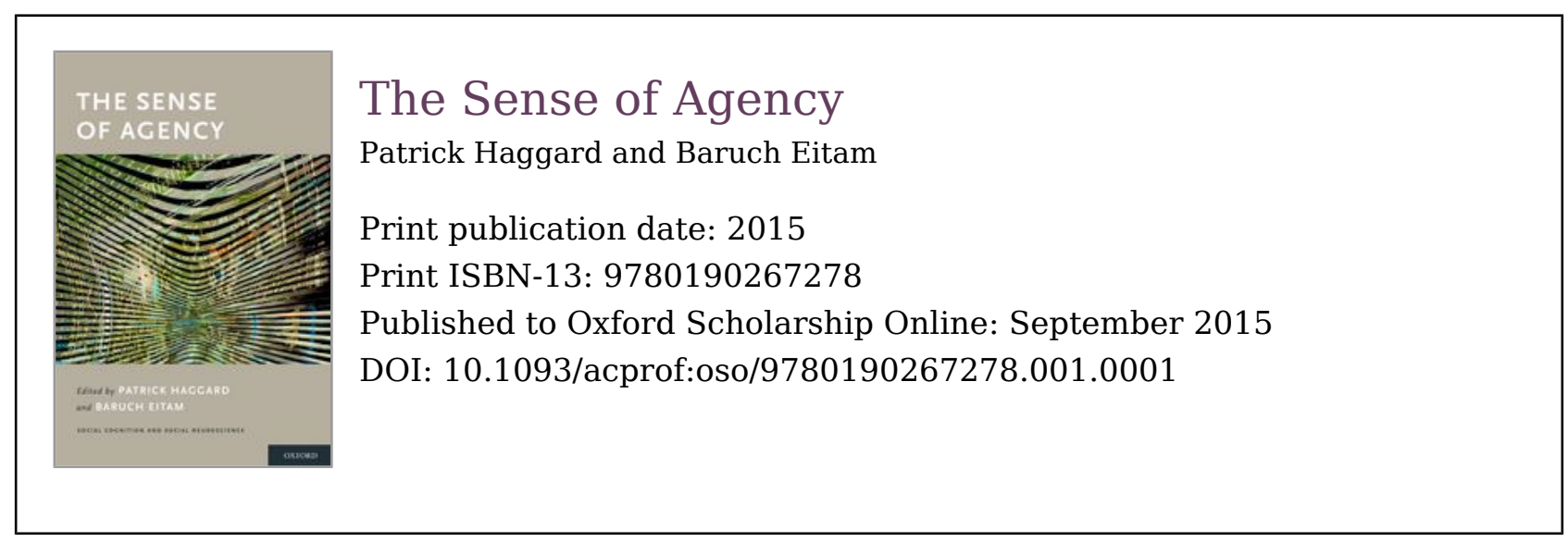

\section{Action Generation, Intention, and Agency in Motor and Body Awareness Deficits}

\author{
Anna Berti \\ Francesca Garbarini \\ Lorenzo Pia \\ DOI:10.1093/acprof:oso/9780190267278.003.0017
}

\section{Abstract and Keywords}

The dramatic impairment of motor and body awareness, often observed in brain-damaged patients, can shed light on the mechanisms implicated in the emergence of conscious experience of the self as an acting body. This chapter first reviews evidence that shows, in patients affected by anosognosia for hemiplegia, how the activation of normal intentional process can give rise to false beliefs of movement when predictive models of motor behavior cannot be confronted with sensory feedbacks, due to damage of motor control systems. In brain-damaged patients with motor and somatosensory deficits, puzzling cognitions and behaviors can 


\section{Action Generation, Intention, and Agency in Motor and Body Awareness Deficits}

sometimes be observed where patients can misidentify other people's limbs as their own, showing a pathological embodiment $(E+)$ of others' body parts (a condition called somatoparaphrenia). The chapter notes that in this condition the sense of agency and the intentional attitude can be "normally" transferred to the movements of another's limbs.

Keywords: impairment, brain damage, anosognosia for hemiplegia

\section{Introduction}

Interaction with other individuals and with the environment is mediated by motor actions through which people try to achieve their goals and purposes. Action is generated through a chain of neurobiological events that is often not available to consciousness, although we are usually aware of moving (or not moving) different parts of our body. Therefore, one has the conscious knowledge that, for instance, one's hand is moving, as well as intentional attitudes, defined as the felt urge to make a movement that precedes the actual execution of a specific motor act. We also feel a strong sense of controlling our own actions (usually indicated as "sense of agency"), and that our own body performs those actions. The normal integration between the different aspects of conscious motor control and the feeling of body ownership (i.e., the experience that bodily states are so clearly and inexorably "mine") are considered to be crucial for the building up of a coherent sense of identity. Therefore, it has been proposed that one of the most fruitful ways to refer to the conscious self is to conceive it as intimately related to the concept of body "as source or power to action, i.e. as the variety of motor potentialities that define the horizon of the world in which we live" (Gallese \& Sinigaglia, 2010). Accordingly, in the present chapter, we shall review some studies that investigate which processes are (p.372) critical when people have beliefs about moving parts of their bodies, and which kind of body experiences are involved in shaping and/or modeling the various senses of motor self-awareness (see also Tsakiris, Chapter 10 of this volume). Neurophysiological and neuropsychological evidence suggest that the neural bases of the different kinds of self-awareness can be kept relatively

\footnotetext{
Page 2 of 25
} 


\section{Action Generation, Intention, and Agency in Motor and Body Awareness Deficits}

distinct and are discretely organized (Spinazzola et al., 2008). The relation between them therefore needs to be clarified in order to capture the real nature and structure of the self.

\section{Agency and Intention}

The sense of agency can be defined as the feeling that I am the one who generates a willed action. The relation between agency and intention seems to be strong: the experience of being in control of one's own movements, and of their effects, is stronger when there is an explicit intentional selection of a motor action than when the same movements are externally generated by a mechanical device or by a transcranial magnetic stimulation (TMS)-induced stimulation of the motor cortex (Haggard \& Clark 2003). For instance, in a recent study, healthy subjects pressed a button, which caused a tone to occur on some trials. Their task was to judge the time they became aware of either pressing the key, or of hearing the tone. When the key press caused the tone, participants judged their key press as occurring later, and the tone earlier, than when the two events occurred alone. The effect did not occur when an involuntary movement (caused by stimulating the motor cortex using TMS) was followed by a tone, or when subjects judged the timing of two causally related external sensory events. This "temporal attraction" strongly suggests that there is a tight link between our experience of agency and motor intentionality. Other evidence confirming how the sense of agency is modulated by motor intentionality comes from brain-damaged patients affected by an unusual form of limb misattribution and will be discussed later in this chapter (see E+ patients).

\section{Intention and Motor Awareness}

The relation between conscious intention and motor awareness has been studied in different areas of psychology and cognitive neuroscience. In a seminal study, Libet and coworkers (Libet et al., 1983) showed that although the awareness of the intention to move (the "W judgment") anticipates the actual movement by about 200 milliseconds, it follows by hundreds of milliseconds (instead of preceding) the electrophysiological preparatory activity related to movement

\footnotetext{
Page 3 of 25
}

PRINTED FROM OXFORD SCHOLARSHIP ONLINE (www.oxfordscholarship.com). (c) Copyright Oxford University Press, 2015. All Rights Reserved. Under the terms of the licence agreement, an individual user may print out a PDF of a single chapter of a monograph in OSO for personal use (for details see http://www.oxfordscholarship.com/page/privacy-policy). Subscriber: 


\section{Action Generation, Intention, and Agency in Motor and Body Awareness Deficits}

(readiness potential), usually registered on the scalp over the supplementary motor area. These results strongly suggested that the conscious experience of (p.373) intentionality is a consequence of brain activity related to the process of programming and selecting the correct movements for action (Haggard \& Eimer, 1999; Libet et al., 1983). Libet and coworkers also asked their subjects to estimate the time at which they became aware of executing a voluntary movement ("M judgment," or motor awareness). Participants' timing of the $\mathrm{M}$ moment followed the timing of the $\mathrm{W}$ moment, as expected. But, surprisingly, it precedes, instead of follows, the actual initiation of the movement by 50 to 80 milliseconds. This outcome suggests that motor awareness emerges before any sensory feedback reaches the brain, thus showing that the feeling that a movement is produced is not linked in an absolute way to the feedback coming from the moving muscles and joints. Although counterintuitive, these results are consistent with other studies that showed that the sensations associated with the actual execution of movements could be unnecessary for the construction of movement awareness. Similarly, Fourneret and Jeannerod (Fourneret \& Jeannerod, 1998) concluded that we are aware of the movement we intend to perform, rather than of the movement we actually produce. Therefore, although proprioception and vision are fundamental aspects of our capacity of judging the course and the consequences of a motor event, motor awareness is somehow independent from their operations, but is strictly related to intentionality. Blakemore and colleagues (Blakemore \& Frith, 2003) suggested that motor awareness must correspond to some neural signal that (a) precedes the movement, (b) follows the development of a conscious intention, and (c) is formed prior to the processing of sensory feedback. They proposed a model of how the control of motor systems relates to various forms of awareness ("the comparator model"; Haggard, 2005; Wolpert et al., 1995). According to the model, once the appropriate motor commands are selected for the execution of the appropriate action, a prediction of the sensory consequences of the movement is formed and would be compared with the feedbacks associated with the actual execution of the intended movement. This prediction is the signal upon which motor

\footnotetext{
Page 4 of 25
} 


\section{Action Generation, Intention, and Agency in Motor and Body Awareness Deficits}

awareness is constructed. Note that because this signal precedes, instead of follows, sensory feedback, it is the ideal candidate for being the basis for Libet's participants' $M$ judgment. Moreover, it is also consistent with Fourneret and Jeannerod's (1998) conclusion. Data on brain-damaged patients confirm this model and shall be discussed later in the chapter.

\section{Motor Cognition and Body Ownership}

As mentioned earlier, a fundamental component of the conscious feeling of moving is the sensation that we are acting with part of our own body (p.374) (see also Tsakiris, Chapter 10 of this volume). Indeed, normal functioning of processes underlying intentional actions and the veridical construction of motor awareness entail a normal sense of body ownership (Tsakiris \& Haggard, 2005). The processes that are involved in the construction of normal body awareness are, however, far from being clear. It has been suggested that several different components contribute to our feeling of body ownership. According to some views, conscious, top-down, cognitive representation that also incorporates semantic knowledge about the body are thought to interact with unconscious, bottom-up, dynamic representation that relies on proprioceptive information from the muscles, joints, and skin (Head \& Holmes, 1911). This latter representation is used to control posture and to enable and shape motor actions. The coherent sense of body ownership that would emerge from these representations can be, however, affected by different experimental interventions that manipulate the sensory-motor state of the body. For example, synchronous touches of a visible rubber hand and the participants' hand (hidden from sight) produce the sensation of feeling the touch on the rubber hand, as well as a compelling feeling of ownership of that hand (the "rubber hand illusion"; e.g., (Botvinick \& Cohen, 1998; Costantini \& Haggard, 2007; Ehrsson et al., 2004; Farnè et al., 2000; Longo et al., 2008; Tsakiris \& Haggard, 2005). This illusion exploits the brain's predilection for integrating congruent tactile, visual, and proprioceptive stimuli, even when they are in contrast with one another. Recent studies also demonstrated that we may actively incorporate tools in

\footnotetext{
Page 5 of 25
}

PRINTED FROM OXFORD SCHOLARSHIP ONLINE (www.oxfordscholarship.com). (c) Copyright Oxford University Press, 2015. All Rights Reserved. Under the terms of the licence agreement, an individual user may print out a PDF of a single chapter of a monograph in OSO for personal use (for details see http://www.oxfordscholarship.com/page/privacy-policy). Subscriber: 


\section{Action Generation, Intention, and Agency in Motor and Body Awareness Deficits}

our body representation, so that the tool changes both our perception of body extension (Sposito et al., 2012) and our mapping of the external world (Berti \& Frassinetti, 2000; Longo \& Lourenco, 2006). Interestingly, these studies showed that "tool embodiment" was present only when the tool was used for performing a voluntary action, suggesting that only intentional tool use shapes one's own body schema.

Accordingly, we shall show the flip side of this principle and present evidence that altered feeling of body ownership directly affects motor cognition. The studies that we shall review in this chapter aim at clarifying the relation between agency, intention, and body ownership from the point of view of pathological behavior, observed in brain-damaged patients with dramatic impairment of motor and body awareness.

\section{Motor Awareness Deficits and Their Relation with Agency and Intention}

In this part of the chapter, the relation between motor awareness, motor intention, and agency will be discussed referring to recent experimental studies (p.375) in braindamaged patients affected by anosognosia for hemiplegia (AHP) and motor neglect who, in different ways, deny their contralesional motor disorders.

An important contribution for the understanding of the neural bases of motor awareness comes from the study of a pathological condition in which movement awareness is dramatically impaired. AHP patients suffering from right-brain damage develop a paresis of the left side of the body but obstinately deny their motor deficit, and when asked to move their paralyzed limb they claim to have performed the action required by the examiner (see Bottini et al., 2010; Fotopoulou, 2012; Orfei et al., 2007; Pia et al., 2004; Spinazzola, Bellan, Pia, \& Berti, 2014). It has been proposed that AHP might be explained as a domain-specific disorder of motor control (Berti \& Pia, 2006; Gold et al., 1994; Jenkinson \& Fotopoulou, 2010; Spinazzola et al., 2008).

Several imaging studies of intact brains show that the cortical network for conscious awareness of action overlaps with that for control of movement (e.g., (Desmurget \& Sirigu, 2009).

\footnotetext{
Page 6 of 25
} 


\section{Action Generation, Intention, and Agency in Motor and Body Awareness Deficits}

Accordingly, it has been demonstrated that AHP follows from brain damage to the same cortical network responsible for motor monitoring. This network is located in the lateral premotor and insular cortex (Berti et al., 2005; Fotopoulou et al., 2010; Garbarini et al., 2013b; Karnath et al., 2005; Moro et al., 2011; Pia et al., 2013b; Vocat et al., 2010). Consequently, the well-established framework of a forward model of normal motor control (Blakemore \& Frith, 2003; Wolpert et al., 1995) has been employed to predict the pattern of intact and impaired neurocognitive mechanisms, pinpointing the distorted motor awareness of AHP patients. As already mentioned, the model posits that, when a subject has the intention to move and the appropriate motor commands are selected and sent to the appropriate motor areas, a prediction (forward model) of the sensory consequences of the movement itself is formed based on the efference copy of the programmed motor act. This would be subsequently matched (by a comparator system) with the actual sensory feedback (see also Gold et al., 1994). The efference copy is the signal from which motor awareness is constructed. This model has two important implications. First, motor awareness would, counterintuitively, precede movement execution, instead of following it. This entails that whenever a sensory prediction is formed, motor awareness emerges before the availability of any sensory feedback. Second, motor awareness is evaluated against the sensory feedback by the operation of the comparator system that, among other functions, can differentiate between movement/no-movement conditions. Within this framework, it has been proposed that, in AHP patients, damage to the comparator processes would alter the monitoring of voluntary actions, thus impairing the ability to distinguish between movement and no-movement states. The (non-veridical) feeling of movement in AHP would then arise from an intact (p.376) motor intentionality, assisted by the normal activity of the brain structures that implement the intention-programming system (Berti \& Pia, 2006; Berti et al., 2007; Garbarini et al., 2012; Pia et al., 2013b; Spinazzola et al., 2008). Several studies clearly demonstrated preserved movement intentionality in AHP patients; first, AHP patients may show normal proximal muscle electrical activity in the affected side when they believe they are moving the plegic 


\section{Action Generation, Intention, and Agency in Motor and Body Awareness Deficits}

limb (Berti et al., 2007; Hildebrandt \& Zieger, 1995). Second, their subjective experience of willed actions is strictly related to their preserved intentionality. For instance, AHP patients falsely detect the movement of their plegic arm when they intend to move it, but do not detect movement when they do not have intention (Fotopoulou et al., 2008). Recently, we directly demonstrated that the motor programs for the affected limbs of AHP patients are identical to those that govern normal movement execution, implying no deficit in generating motor intentions (Garbarini et al., 2012; Pia et al., 2013b). We did this by taking advantage of the difficulties of bimanual motor tasks. When both hands move simultaneously, strong coupling effects arise and neither hand is able to perform independent actions successfully (see Swinnen, 2002, for a review). This conflict stems largely from internal motor programming rather than from the on-line feedback coming from movement execution (Drewing et al., 2004; Franz \& Ramachandran, 1998; Spencer et al., 2005; but see Mechsner et al., 2001, for a different point of view). We expected that, in AHP patients, these bimanual interference effects should be present, because motor programs and sensory predictions are both present, despite the absence of movement and sensory feedback for the affected hand. Using bimanual motor tasks, in which AHP patients were asked to simultaneously perform movements with both hands, we found that the movements of the intact hand were influenced by the intended movements of the paralyzed hand, although these movements were, of course, not actually executed. This influence produced both spatial (Garbarini et al., 2012) and temporal (Pia et al., 2013b) coupling effects, comparable to those found in healthy subjects actually performing bimanual tasks. Examples of spatial bimanual coupling, for both healthy subjects and AHP patients, are shown in Figure 17.1 A and B. Recently, it has been showed that the spared intention-programming system can affect other distal kinematic parameters of the healty hand as grip aperture (Piedimonte, in press). These findings in AHP patients suggest that their motor awareness may be constructed from a normal intentional process, even in absence of movement execution. 


\title{
Action Generation, Intention, and Agency in Motor and Body Awareness Deficits
}

\begin{abstract}
An intriguing contrast to AHP was provided by patients without hemiplegia who were affected by the distinct neurological syndrome of motor neglect (MN). Indeed,

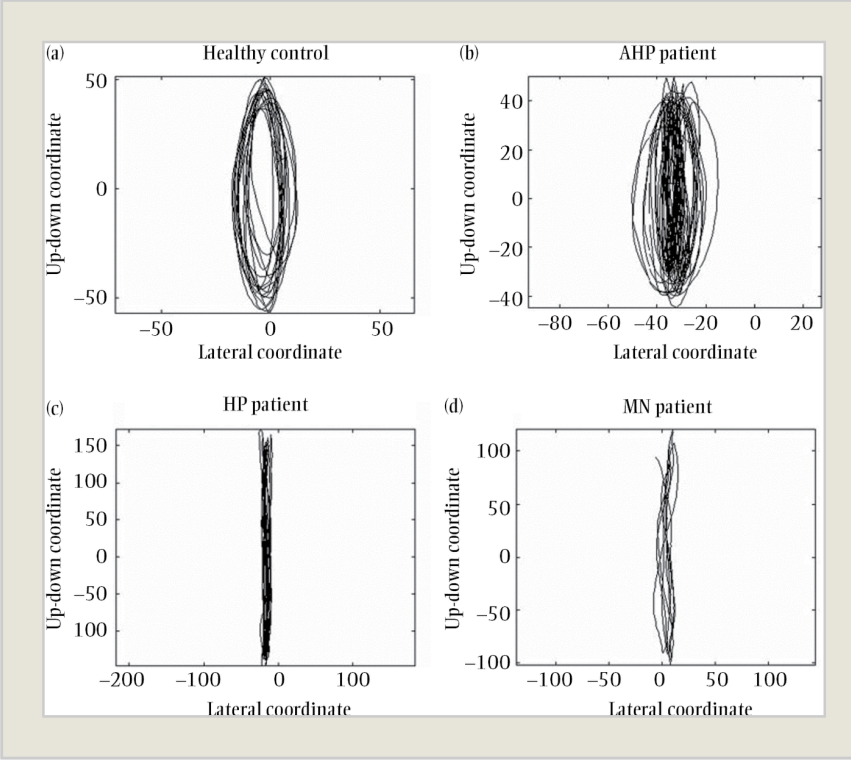
$\mathrm{MN}$ can, in some ways, be considered "the neurological opposite" of AHP. Sometimes described as "pseudo-hemiplegia" (Laplane \& Degos, (p.377) (p.378) 1983), and often interpreted as the consequence of a damage to intentional motor circuits (Berti et al., 2007; Gold et al., 1994), MN is characterized by underutilization of the contralesional limb in the presence of normal strength, reflexes, and sensibility and thus preserved potential for actual movement on the affected side. Crucially, when $\mathrm{MN}$ patients are asked to perform bimanual movements, they only perform ipsilesional hand movements (Laplane \& Degos, 1983), even though (unlike AHP cases) they are actually capable of moving the contralesional hand. However, unlike AHP patients or normal controls, MN cases did not show any coupling effect (Garbarini et al., 2012). This pattern was also obtained with HP cases (without anosognosia), even though MN patients differ in being physically capable of moving the contralesional limb (see Figure 17.1 C and D). The MN cases provide an interesting contrast to the AHP cases. The former are non-plegic but apparently lacking intention/ planning, whereas the latter are plegic but still maintain intentions/plans for the affected hand.

Although AHP and MN are different in terms of motor intention and motor planning, it has been recently demonstrated that they are both characterized by lack of awareness for the motor impairment (Garbarini et al., 2013a).
\end{abstract}




\section{Action Generation, Intention, and Agency in Motor and Body Awareness Deficits}

This finding
can be
explained
within the
computational
model of
motor control
described
earlier. More
precisely,
denial
behavior in
AHP patients
may be due to
direct damage
to the
comparator
system, which
cannot detect
the mismatch
between the
intended but
unexecuted
action. The
evident
feeling of
movement
that AHP
patients
(erroneously)
experience
would then
arise from
intact
structures
implementing
motor
intentionality.
Conversely, a
intentioit at the

Page 10 of 25
Figure 17.1 A modified version of a

Circles-Lines task (Franz et al., 1991) was used to investigate the spatial bimanual coupling. Participants were asked to draw circles and lines, either performing unimanual drawing movements-vertical lines with the right (intact) hand-or bimanual movements-vertical lines with the right hand and simultaneously, circles with the left (paralyzed) hand. In normal subjects, we know that the trajectory of the right hand drawing lines tends to assume an oval shape (i.e., more spatial error) when the other hand must concurrently draw circles, compared to unimanual conditions, indicating that the motor programs for the hand drawing circles can affect the motor programs of the hand drawing lines, to produce a bimanual interference effect (Franz et al., 1991). We predicted that, if patients with anosognosia for hemiplegia (AHP) do intend and plan movements with their paralyzed hand, the lines drawn by the intact hand should become more oval when the patients are requested to draw a circle concurrently with their plegic hand, as found in normal subjects.

Different results were expected in hemiplegic patients fully aware of their motor deficits (hemiplegic patients without AHP) and in patients without hemiplegia who do not intend/plan contralesional movements in bimanual situations (motor neglect [MN] patients). In Figure 17.1, examples of subjects' right-hand trajectory in the crucial bimanual Circle-Line condition are shown. Note the increased ovalization for healthy controls (A) and for hemiplegic patients with AHP (B), but not for hemiplegic patients without AHP (C) or patients with $\mathrm{MN}$ (D). 


\section{Action Generation, Intention, and Agency in Motor and Body Awareness Deficits}

programming level can explain MN. Here, brain damage causes an inability to form motor intentions; if the intention to move is defective, motor planning is prevented. Because the comparator, although working properly, does not receive any information about movement planning, it cannot interpret the lack of movements as aberrant (Gold et al., 1994). Therefore, MN patients are completely unaware of the lack of any execution, not because of a direct damage to the comparator system, as it has been proposed for AHP patients, but because they cannot discover the abnormality of their behavior, since they do not attempt to make any movement.

In summary, the evidence described here, in AHP patients, suggests that conscious intention to move can be experienced without actual movement. The cerebral areas that seem to be involved in this process are the mesial-frontal and posteriorparietal areas (spared in AHP patients) for the intentional component of the motor act, and the premotor and insular cortices (damaged in AHP patents) for the process of construction of motor awareness. It seems logical that the sense of agency, the feeling that "I" am the one who is controlling the movements, should be related to the presence of an ongoing action (p.379) (Haggard, 2005). However, pathological conditions in which the presence of actions is dissociated from both the sense of agency and conscious intention have been described. For instance, in the anarchic hand syndrome, patients perform actions coherent in respect to environmental stimuli that do not correspond to the patient's conscious free will (see Rowe \& Wolpe, Chapter 18 of this volume). These patients have intact motor awareness and motor function, but they feel that it is not their "self" that is in charge of the decision of moving. In contrast, anosognosic patients not only believe that their left limbs can move, but they are always convinced that they are the ones who move the arm. In other words, they never doubt their authorship once they have programmed a movement, despite the impossibility of performing it. Moreover, they seem to have intact, although decontextualized, motor intention: they try to make movements with the paralyzed limbs, both spontaneously and under request. This picture suggests that, although the sense of agency can be present without any

Page 11 of 25

PRINTED FROM OXFORD SCHOLARSHIP ONLINE (www.oxfordscholarship.com). (c) Copyright Oxford University Press, 2015. All Rights Reserved. Under the terms of the licence agreement, an individual user may print out a PDF of a single chapter of a monograph in OSO for personal use (for details see http://www.oxfordscholarship.com/page/privacy-policy). Subscriber: Universita di Torino; date: 13 June 2016 


\section{Action Generation, Intention, and Agency in Motor and Body Awareness Deficits}

ongoing motor activity, it is tightly linked to the feeling of intention to move, even when that feeling is constructed on pathological beliefs.

\section{Body Awareness Deficits and Their Relation with Agency and Intention}

In this section of the chapter, the relation between motor awareness, motor intention, and agency will be discussed, referring to recent experiments carried out in some patients who, despite being aware of their contralesional sensorymotor deficits, believe that someone else's limb is attached to them.

In right-brain-damaged patients with motor and somatosensory impairments, a body awareness deficit can sometimes be observed (a disturbance called somatoparaphrenia). Patients may feel a sense of strangeness toward contralesional limbs that may be felt as separated from the patients' body. The more frequent manifestation of this disorder is characterized by a sense of disownership, which is the delusional belief that the contralesional limbs do not belong to one's own body but to another person (Jenkinson, Haggard, Ferreira, Fotopoulou, 2013; Vallar \& Ronchi, 2009). Recently, an anatomical account of somatoparaphrenia as delusion of disownership has been proposed (Gandola et al., 2012), suggesting a crucial involvement of white matter and subcortical gray structures (thalamus, basal ganglia, and amygdala). Recently we observed the case of a somatoparaphrenic patient who attributed his contralesional arm to his brother. He claimed that when the nurses took the blood from his arm they were actually taking the blood from his brother's arm and expressed his unhappiness about the nurses needlessly intervening to take blood from his brother's arm, when his brother was demonstrably healthy. Once the somatoparaphrenia recovered, the patient not only regained consciousness of his own (p.380) left arm, but also remembered his delusional behavior. Crucially, he reported having previously held the false belief that his left arm belonged to his brother (unpublished case). 


\section{Action Generation, Intention, and Agency in Motor and Body Awareness Deficits}

The possibility that an opposite behavior exists, that is, patients who misidentify other people's limbs as if they were their own, has been rarely considered. However, in recent studies (Garbarini \& Pia, 2013; Garbarini et al., 2013b; Garbarini, Fornia, et al., 2014; Garbarini, Fossataro, et al., 2015; Pia et al., 2013a), we observed this behavior in a sample of hemiplegic and/or hemianesthesic patients. While they did not explicitly deny that their contralesional (left) limbs belonged to themselves (as in the somatoparaphrenic delusion of disownership), they claimed that the examiner's left hand was their own whenever it was positioned, in egocentric coordinates, on the table next to their real left hand. This delusion of ownership, although resembling the "rubber hand illusion" (Botvinick \& Cohen, 1998), was spontaneous and not induced by any experimental procedure. Patients treated and cared for the experimenter's left arm as if it were their own, showing a consistent embodiment of an alien hand in their own body schema. Because of this pathological embodiment, we named them E+ patients. Interestingly, this phenomenon occurs not only with a static alien hand but also when movements are present, that is, when the examiner moved his or her left hand, patients claimed that they were moving their own (paralyzed) hand.

It is worth noting that, as mentioned earlier, at the time of testing $\mathrm{E}+$ patients did not show any explicit form of disownership, never spontaneously reporting delusional beliefs about the contralesional body parts. Accordingly, when only their left hand was present, they correctly identified it as their own left hand. However, when both the own and the alien hands were present, not only did they misidentify the alien hand as their own, but they also identified their own left hand as alien, affirming that it belonged to someone else, thus showing an explicit sense of disownership. This suggests that the two delusional behaviors (disownership of the own hand and ownership of an alien hand) may coexist in the same patients, though directed at different objects (see also Kammers et al., 2011; Moseley et al., 2008, for similar results on the rubber hand illusion). The fact that sometimes the disownership behavior is immediately evident while another time it emerges only as a consequence of the alien hand

Page 13 of 25

PRINTED FROM OXFORD SCHOLARSHIP ONLINE (www.oxfordscholarship.com). (c) Copyright Oxford University Press, 2015. All Rights Reserved. Under the terms of the licence agreement, an individual user may print out a PDF of a single chapter of a monograph in OSO for personal use (for details see http://www.oxfordscholarship.com/page/privacy-policy). Subscriber: 


\section{Action Generation, Intention, and Agency in Motor and Body Awareness Deficits}

misattribution may suggest that these two forms of body unawareness lay on a continuum, possibly characterizing different phases (acute/sub-acute/chronic) of the disease or different intensity of the deficit. Furthermore, the E+ patients' lesion pattern seems consistent with that described in previous studies on neural correlates of the delusion of disownership (Gandola et al., 2012; Garbarini et al., 2013b).

(p.381) Starting from the clinical description of these cases, different questions can be addressed: Can we really incorporate into our body schema the body parts of others, altering our sense of body ownership? In other words, is "my" body schema fixed and immutable and does it correspond only to "me," or can part of others' bodies become part of my sensory-motor representations? And if the answer to the last question is positive, does this incorporation influence how we consciously perceive our body? Body image and body perception would therefore be extended to others' body parts, so that the stimulation of embodied alien hand may elicit subjective sensation specific to our own body. Furthermore, to what extent, given the tight link between body and motor representations, does an altered sense of body ownership affect patients' intentional attitude and their motor awareness and sense of agency? To answer these questions, we conducted a series of experiments, some of them still in progress, aiming at verifying the consequences of the altered body awareness on the patients' sensory-motor parameters.

We investigated the somatosensory domain (Pia et al., 2013a; Garbarini et al., 2014), reasoning that the pathological embodiment observed in $\mathrm{E}+$ patients is an ideal condition to examine whether tactile/pain sensations can be "transferred" to an alien arm subjectively experienced as own. Patients (with and without the delusion) and healthy controls were tested with a pinprick protocol to assess pain perception. In the own hand condition, participants placed their arms on a table and the hand dorsum (either of the right or of the left hand) was stimulated. In the alien hand condition, the co-experimenter's left or right arm was placed alongside the participants' left or right arm, respectively, and the left or right co-experimenter's hand dorsum was stimulated. In both conditions, participants

Page 14 of 25

PRINTED FROM OXFORD SCHOLARSHIP ONLINE (www.oxfordscholarship.com). (c) Copyright Oxford University Press, 2015. All Rights Reserved. Under the terms of the licence agreement, an individual user may print out a PDF of a single chapter of a monograph in OSO for personal use (for details see http://www.oxfordscholarship.com/page/privacy-policy). Subscriber: Universita di Torino; date: 13 June 2016 


\section{Action Generation, Intention, and Agency in Motor and Body Awareness Deficits}

had to rate the perceived sensation on a Likert scale. We hypothesized that, if the false belief of owning an alien arm is not a mere verbal confabulation but, rather, the result of a profound embodying mechanism that affects the E+ patients' sensory processing, then this should paradoxically produce a feeling of pain not only in the own condition, when the stimuli are actually applied on the patients' own hand, but also in the alien condition, when stimuli are applied onto the coexperimenter's left hand. The results of a first behavioral experiment (Pia et al., 2013) confirmed these predictions, showing that a body part of another individual can become, in a pathological condition, so deeply embedded in one's own somatosensory representation to have a consistent effect on the subjective sensation of pain. Furthermore, in a second experiment where the skin conductance was recorded during noxious stimulations (either to the own or the alien hand), we demonstrated that the alien (embodied) hand can be able to elicit physiological reactions specific to the own hand (Garbarini et al., 2014).

(p.382) In these patients, we also investigated the motor domain (Garbarini et al., 2013b; Garbarini et al., 2015) and we asked whether, once an alien hand is embodied into the patient's body schema, its representation can affect motor production and motor control as if it actually belonged to the patients. E+ patients were asked to execute a modified version of a bimanual Circles-Lines task (Franz \& Ramachandran, 1998; Garbarini et al., 2012; Garbarini et al., 2013; Garbarini et al., 2015; Piedimonte et al., 2014) in which they had to draw lines with their intact hand while watching an alien hand performing circles, either in an egocentric position, that is, congruent with the position of the patients' left hand, or in an allocentric non-congruent position, that is, positioned opposite the patient. As in the previous experiment, the crucial aspect was that in the congruent condition $\mathrm{E}+$ patients misidentified the alien hand as their own, while in the non-congruent condition patients' recognize the alien hand as belonging to the co-experimenter. We hypothesized that, if the delusion of ownership arises from an abnormal embodiment process that automatically triggers the intention-programming processes for the own hand, when the alien hand drew circles in the 


\section{Action Generation, Intention, and Agency in Motor and Body Awareness Deficits}

egocentric condition, the lines drawn by the E+ patients' intact hand should become ovalized (coupling effect), as in normal individuals actually performing the bimanual task (see Figure 17.1). The E+ patients' results verified these predictions, showing a clear coupling effect in the alien congruent (egocentric) condition, in which patients claimed having performed circles with their own left hand. It is important to note that, in the same condition, neither healthy controls nor hemiplegic patients without embodiment showed any coupling effect. This suggests that simply looking at a hand drawing circles is not sufficient to induce line ovalization.

In a different experiment (Garbarini et al., 2015), always related to the motor domain, we investigated in E+ patients the effect of tool-use training on length representation of their contralesional forearm. We know from the literature that an active tool-use can reshape one's own body schema, extending peripersonal space and modulating the representation of related body parts (e.g., Sposito et al., 2012). In our task, an alien hand performed the tool-use training, acting either in a body-congruent position (aligned with the patients' shoulder; where the pathological embodiment occurs) or in a nocongruent position (misaligned with the patients' where the pathological embodiment does not occur). Coherently, only in the body-congruent condition, when patients were convinced to perform the tool-use training with their own paralyzed arm, a significant overestimation effect was found.

These findings clearly showed that a profoundly altered sense of body ownership affects both motor awareness ( $\mathrm{E}+$ patients, usually aware of their motor impairment, were convinced that their left hand was moving) and sense of agency ( $E+$ patients ascribed the alien movements to themselves), by directly (p.383) modulating either the action execution (E+ patients showed an interference/coupling effect very similar to those found in healthy subjects actually performing bimanual Circles-Lines task) or the body length representation ( $\mathrm{E}+$ patients showed an overestimation effect very similar to those found in healthy subjects actually performing the tool-use training). It is important to emphasize that E+ patients, although similar to anosognosic patients in relation to the

Page 16 of 25

PRINTED FROM OXFORD SCHOLARSHIP ONLINE (www.oxfordscholarship.com). (c) Copyright Oxford University Press, 2015. All Rights Reserved. Under the terms of the licence agreement, an individual user may print out a PDF of a single chapter of a monograph in OSO for personal use (for details see http://www.oxfordscholarship.com/page/privacy-policy). Subscriber: Universita di Torino; date: 13 June 2016 


\section{Action Generation, Intention, and Agency in Motor and Body Awareness Deficits}

presence of hemiplegia, are, however, behaviorally different. Indeed, in everyday situations they acknowledge their motor deficit, therefore showing normal motor awareness and motor monitoring. However, the presence of an alien hand in an egocentric position crucially affects their sensory-motor processes and motor consciousness. It is still to be clarified how this can happen. In the rubber hand illusion the discrepancy between visual, tactile, and proprioceptive input is resolved by the brain with the transient incorporation of the rubber hand into the (normal) subjects' body schema. We may speculate that when the representation of the contralesional hand is partially impaired or made fragile by the brain damage, as in E+ patients, the brain solves the incoherence of the "mutilated" body representation by automatically incorporating an alien hand (when positioned in the egocentric space) so to regain consistency and functionality. As a consequence, the alien hand becomes part of the patients' body representation, and starts to have an impact on the patients' sensory-motor processes. This, in turn, modulates motor awareness and the sense of agency. Note that the alien hand embodiment is fully dependent on seeing the alien hand. However, vision is not fundamental per se, but is so only if the alien hand is in a position that is congruent with the patients' body schema. Only in this condition does the image of the hand trigger a reconstruction of the patients' body representation. The Circles-Lines task, employed in this experiment, allowed us to describe to what extent this modulation is effective. Our results showed that body parts belonging to other people can be so fully incorporated into one's own body schema as to imply sensory-motor consequences on one's own action execution.

\section{Conclusion}

In this chapter we have discussed the relation between different components of motor cognition, capitalizing on recent findings obtained in brain-damaged patents. Pathology, integrated with data from healthy subjects, is fundamental for breaking with the accepted depiction of some mental processes. In particular, the traditional views on agency, intention, and motor awareness are challenged by the patients' behavior described in the previous paragraphs. First,

Page 17 of 25 


\section{Action Generation, Intention, and Agency in Motor and Body Awareness Deficits}

it has been shown that under particular conditions, as occurs in anosognosic patients, the presence of normal motor intentionality is sufficient to construct (p.384) motor awareness for a given action in absence of any real movement execution. This confirms that full consciousness of a volitional motor act implies the activation of a cascade of motor events that construct motor awareness based on a combination of intention and prediction. Previous studies in healthy subjects had already raised this possibility. However, our results offer strong further support that we are aware of what we intend to do instead of what we really do (see also Fourneret \& Jeannerod, 1998) Specifically, we found counterintuitive but objective consequences for the motor behavior of the unaffected hand due to programmed but not executed movement of the paralyzed hand (in AHP patients). In the AHP patients, the sense of agency seems to be "normal," or at least conforms with the new body condition, insofar as the movement that they believe was executed is recognized as their own, that is, related to their own will. It is difficult to identify the precise neural signal denoting the sense of agency. However, it seems to be constructed from a "normal" integration of both intentionality and motor awareness, despite absence of actual movement: I have the intention to move, I feel that I move, therefore I am the one who is controlling the movements.

Even more intriguing is the relation between voluntary action, body sensation, and body ownership, which is at the core of humans' sense of self. Here we described a pathological condition in which brain-damaged patients, under particular constraints, automatically embodied other people's arms. Whatever anomaly leads to such a profound alteration of the sense of body ownership may be the crucial aspect of this false belief and its objective effects on the sensory-motor awareness of the patients. In absence of any kind of voluntary action, humans' body ownership is built up through the interaction between afferences (e.g., proprioception and vision) and preexisting representations of the body. This integration, in health, allows people to distinguish their own bodies from those of others (and from the surrounding world). When a voluntary action is performed, efferent information is added to

Page 18 of 25 


\section{Action Generation, Intention, and Agency in Motor and Body Awareness Deficits}

this process. Hence, the match between efferences, afferences, and pre-existing body representations allows discrimination between our own actions and the actions of other people. Both afferences integration and efferent processes are affected in E+ patients, who, once they have embodied the "alien" arm, feel sensation on it (in absence of any real stimulation or afference) and feel that they move it (in absence of any real movement of their own limb, again in absence of any afferent feedback). Viewing the embodied alien arm moving seems to trigger the same motor cascade that leads from conscious intention to motor awareness, in healthy participants and in AHP patients. The system seems to respond to the moving alien arm as if the movement were initiated by the patients themselves. Once the cascade is triggered, the sense of agency is affected as well because E+ patients ascribe the observed movement to their own will. Again, (p.385) the sense of agency seems to be the result of a negotiation between intention and motor awareness: I have the intention to move, I feel that I move, therefore I am the one who is controlling the movements.

References

Bibliography references:

Berti, A., \& Frassinetti F. (2000). When far becomes near: remapping of space by tool use. Journal of Cognitive Neuroscience, 12(3), 415-420.

Berti, A., \& Pia, L. (2006). Understanding motor awareness through normal and pathological behavior. Current Directions in Psychological Science, 15(5), 245-250.

Berti, A., et al. (2007). Motor awareness and motor intention in anosognosia for hemiplegia. In P. Haggard, Y. Rossetti, \& M. Kawato (Eds.), Sensorimotor foundations of higher cognition (pp. 17-38). Oxford: Oxford University Press.

Berti, A., et al. (2005). Shared cortical anatomy for motor awareness and motor control. Science, 309(5733), 488-491.

Blakemore, S. J., \& Frith, C. (2003). Self-awareness and action. Current Opinion in Neurobiology, 13(2), 219-224. 


\section{Action Generation, Intention, and Agency in Motor and Body Awareness Deficits}

Bottini, G., et al. (2010). Anosognosia for hemiplegia and models of motor control: insights from lesional data. In G. P. Prigatano (Ed.), The Study of Anosognosia (pp. 363-379). Oxford: Oxford University Press.

Botvinick, M., \& Cohen, J. (1998). Rubber hands 'feel' touch that eyes see. Nature, 391(6669), 756.

Costantini, M., \& Haggard, P. (2007). The rubber hand illusion: sensitivity and reference frame for body ownership. Consciousness and Cognition, 16(2), 229-240.

Desmurget, M., \& Sirigu, A. (2009). A parietal-premotor network for movement intention and motor awareness. Trends in Cognitive Sciences, 13(10), 411-419.

Drewing, K., et al. (2004). Timing of bimanual movements and deafferentation: implications for the role of sensory movement effects. Experimental Brain Research, 158(1), 50-57.

Ehrsson, H. H., Spence, C., \& Passingham, R. E. (2004). That's my hand! Activity in premotor cortex reflects feeling of ownership of a limb. Science, 305(5685), 875-877.

Farnè, A., et al. (2000). Left tactile extinction following visual stimulation of a rubber hand. Brain, 123(11), 2350-2360.

Fotopoulou, A. (2012). Illusions and delusions in anosognosia for hemiplegia: from motor predictions to prior beliefs. Brain, $135,1344-1346$.

Fotopoulou, A., Tsakiris, M., Haggard, P., Vagopoulou, A., Rudd, A., \& Kopelman, M. (2008). The role of motor intention in motor awareness: An experimental study on anosognosia for hemiplegia. Brain, 131, 3432-3442.

Fotopoulou, A., et al. (2010). Implicit awareness in anosognosia for hemiplegia: unconscious interference without conscious re-representation. Brain, 133(12), 3564-3577.

Fourneret, P., \& Jeannerod, M. (1998). Limited conscious monitoring of motor performance in normal subjects. Neuropsychologia, 36(11), 1133-1140.

Page 20 of 25 


\section{Action Generation, Intention, and Agency in Motor and Body Awareness Deficits}

Franz, E. A., \& Ramachandran, V. S. (1998). Bimanual coupling in amputees with phantom limbs. Nature

Neuroscience, 1(6), 443-444.

Franz, E. A., Zelaznik, H. N., \& McCabe, G. (1991). Spatial topological constraints in a bimanual task. Acta Psychologica (Amsterdam), 77(2), 137-151.

Gallese, V., \& Sinigaglia, C. (2010). The bodily self as power for action. Neuropsychologia, 48(3), 746-755.

Gandola, M., et al. (2012). An anatomical account of somatoparaphrenia. Cortex, 48(9), 1165-1178.

Garbarini, F., \& Pia, L. (2013). Bimanual coupling paradigm as an effective tool to investigate productive behaviors in motor and body awareness impairments. Frontiers in Human Neuroscience, 7, 737.

Garbarini, F., et al. (2013a). Dissociations and similarities in motor intention and motor awareness: the case of anosognosia for hemiplegia and motor neglect. Journal of Neurology, Neurosurgery and Psychiatry, 84(4), 416-419.

Garbarini, F., et al. (2013b). Embodiment of an alien hand interferes with intact-hand movements. Current Biology, 23(2), R57-R58.

Garbarini, F., et al. (2012). "Moving" a paralysed hand: bimanual coupling effect in patients with anosognosia for hemiplegia. Brain, 135(5), 1486-1497.

Garbarini, F., Fornia, L., Fossataro, C., Pia, L., Gindri, P., \& Berti, A. (2014). Embodiment of others' hands elicits arousal responses similar to one's own hands. Current Biology, 24(16), R738-9.

Garbarini, F., Dagata, F., Piedimonte, A., Sacco, K., Rabuffetti, M., Tam, F., Cauda, F., Pia, L., Geminiani, G., Duca, S., Graham, S., \& Berti, A. (2013). Drawing lines while imagining circles: neural basis of the bimanual coupling effect during motor execution and motor imagery. NeuroImage, 88, 100122. 


\section{Action Generation, Intention, and Agency in Motor and Body Awareness Deficits}

Garbarini, F., Rabuffetti, M., Piedimonte, A., Solito, G., \& Berti, A. (2015). Bimanual coupling effects during arm immobilization and passive movements. Human Mov Science, $41,114-126$.

Garbarini, F., Fossataro, C., Berti, A., Gindri, P., Romano, D., Pia, L., Della Gatta, F., Maravita, A., \& Neppi-Mòdona, M. (2015). When Your Arm Becomes Mine: Pathological Embodiment of Alien Limbs Using Tools Modulates Own Body Representation. Neuropsychologia, 70, 402-413.

Gold, M., et al. (1994). Anosognosia for hemiplegia: an electrophysiologic investigation of the feed-forward hypothesis. Neurology, 44(10), 1804-1808.

Haggard, P. (2005). Conscious intention and motor cognition. Trends in Cognitive Sciences, 9(6), 290-295.

Haggard, P., \& Eimer, M. (1999). On the relation between brain potentials and the awareness of voluntary movements. Experimental Brain Research, 126(1), 128-133.

Haggard, P., \& Clark, S. (2003). Intentional action: conscious experience and neural prediction. Consciousness and Cognition, 12(4), 695-707.

Head, H., \& Holmes, J. (1911). Sensory disturbances from cerebral lesions. Brain, 34, 102-254.

Hildebrandt, H., \& Zieger, A. (1995). Unconscious activation of motor responses in a hemiplegic patient with anosognosia and neglect. European Archives of Psychiatry and Clinical Neuroscience, 246(1), 53-59.

Jenkinson, P. M., \& Fotopoulou, A. (2010). Motor awareness in anosognosia for hemiplegia: experiments at last! Experimental Brain Research, 204(3), 295-304.

Jenkinson, P. M., Haggard, P., Ferreira, N. C., \& Fotopoulou, A. (2013). Body ownership and attention in the mirror: Insights from somatoparaphrenia and the rubber hand illusion. Neuropsychologia, 51, 1453-1462. 


\section{Action Generation, Intention, and Agency in Motor and Body Awareness Deficits}

Kammers, M. P., Rose, K., \& Haggard, P. (2011). Feeling numb: temperature, but not thermal pain, modulates feeling of body ownership. Neuropsychologia, 49(5), 1316-1321.

Karnath, H. O., et al. (2005). Normalized perfusion MRI to identify common areas of dysfunction: patients with basal ganglia neglect. Brain, 128(Pt 10), 2462-2469.

Laplane, D., \& Degos, J. D. (1983). Motor neglect. Journal of Neurology, Neurosurgery and Psychiatry, 46(2), 152-158.

Libet, B., et al. (1983). Time of conscious intention to act in relation to onset of cerebral activity (readiness-potential): the unconscious initiation of a freely voluntary act. Brain, 106, 623-642.

Longo, M. R., \& Lourenco, S. F. (2006). On the nature of near space: effects of tool use and the transition to far space. Neuropsychologia, 44(6), 977-981.

Longo, M. R., et al. (2008). What is embodiment? A psychometric approach. Cognition, 107(3), 978-998.

Mechsner, F., et al. (2001). Perceptual basis of bimanual coordination. Nature, 414(6859), 69-73.

Moro, V., et al. (2011). Phenomenology and neural correlates of implicit and emergent motor awareness in patients with anosognosia for hemiplegia. Behavioural Brain Research, 225(1), 259-269.

Moseley, G. L., et al. (2008). Psychologically induced cooling of a specific body part caused by the illusory ownership of an artificial counterpart. Proceedings of the National Academy of Sciences of the United States of America, 105(35), 1316913173.

Orfei, M. D., et al. (2007). Anosognosia for hemiplegia after stroke is a multifaceted phenomenon: a systematic review of the literature. Brain, 130(12), 3075-3090.

Pia, L., et al. (2004). The anatomy of anosognosia for hemiplegia: a meta-analysis. Cortex, 40(2), 367-377.

Page 23 of 25

PRINTED FROM OXFORD SCHOLARSHIP ONLINE (www.oxfordscholarship.com). (c) Copyright Oxford University Press, 2015. All Rights Reserved. Under the terms of the licence agreement, an individual user may print out a PDF of a single chapter of a monograph in OSO for personal use (for details see http://www.oxfordscholarship.com/page/privacy-policy). Subscriber:

Universita di Torino; date: 13 June 2016 


\section{Action Generation, Intention, and Agency in Motor and Body Awareness Deficits}

Pia, L., et al. (2013a). Pain and body awareness: evidence from brain-damaged patients with delusional body ownership. Frontiers in Human Neuroscience, 7, 298.

Pia, L., et al. (2013b). Temporal coupling due to illusory movements in bimanual actions: Evidence from anosognosia for hemiplegia. Cortex, 49(6), 1694-1703.

Piedimonte, A., Garbarini, F., Rabuffetti, M., Pia, L., \& Berti, A. (2014). Executed and Imagined Bimanual Movements: A Study Across Different Ages. Developmental Psychology, 50(4), 1073-1080.

Piedimonte, A., Garbarini, F., Rabuffetti, M., Pia, L., Montesano, A., Ferrarin, M., \& Berti, A. (in press). Invisible Grasps: Grip Interference in Anosognosia for Hemiplegia. Neuropsychology.

Spencer, R. M., et al. (2005). Bimanual coordination during rhythmic movements in the absence of somatosensory feedback. Journal of Neurophysiology, 94(4), 2901-2910.

Spinazzola, L., et al. (2008). Modular structure of awareness for sensorimotor disorders: evidence from anosognosia for hemiplegia and anosognosia for hemianaesthesia.

Neuropsychologia, 46(3), 915-926.

Spinazzola, L., Bellan, G., Pia, L., \& Berti, A. (2014).

Unawareness of Motor and Somatosensory deficits after stroke (UMAS): Validity and Reliability of UMAS Questionnaire. Applied Psychology Bullettin, 62(270), 37-58.

Sposito, A., et al. (2012). Extension of perceived arm length following tool-use: clues to plasticity of body metrics.

Neuropsychologia, 50(9), 2187-2194.

Swinnen, S. P. (2002). Intermanual coordination: from behavioural principles to neural-network interactions. Nature Reviews Neuroscience, 3(5), 348-359.

Tsakiris, M., \& Haggard, P. (2005). The rubber hand illusion revisited: visuotactile integration and self-attribution. Journal 


\section{Action Generation, Intention, and Agency in Motor and Body Awareness Deficits}

of Experimental Psychology Human Perception \&

Performance, 31(1), 80-91.

Vallar, G., \& Ronchi, R. (2009). Somatoparaphrenia: a body delusion. A review of the neuropsychological literature.

Experimental Brain Research, 192(3), 533-551.

Vocat, R., et al. (2010). Anosognosia for hemiplegia: a clinicalanatomical prospective study. Brain, 133(12), 3578-3597.

Wolpert, D. M., Ghahramani, Z., \& Jordan, M. I. (1995). An internal model for sensorimotor integration. Science, 269(5232), 1880-1882.

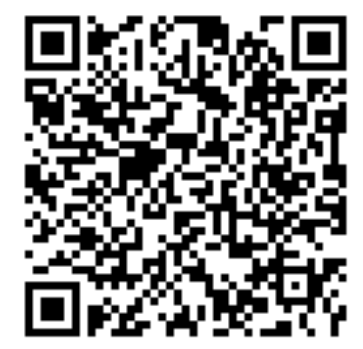

Access brought to you by: Universita di Torino 